\section{NHS CONTRACTS}

Northern Ireland pilots due to start Applications are now open for the first wave of Northern Ireland's pilot process. This is a long-awaited step in anticipation of a new contract for General Dental Services (GDS).

Run by the Health and Social Care Board (HSCB) the pilot will take place in two waves. The first wave is expected to run from September 2014 for 18 months. Pilots will usually include the whole practice and there are expected to be up to five sites in the first wave. The second wave will start at plus six months and will run for 12 months, with up to 15 additional sites.

The British Dental Association (BDA) has welcomed the news of piloting but has serious concerns about the general health of the GDS in the light of the impact of cuts.

Have your say on contract reform The BDA urges dentists to respond to key questions about the progress of contract reforms by the end of July.

A series of reports published earlier this month by the Department of Health (DH) ask dentists for their views on how they should be paid in a changed system and whether the remuneration approach should involve capitation, activity or a blend.

The DH also seeks dentists' views on the clinical philosophy (primary care pathways), the measurement of quality and outcomes, and questions about the practical implications of the current direction of travel for practices and patients.

Dr John Milne, Chair of the BDA's General Dental Practice Committee, said: 'Dentists will have to implement any new system that is adopted so it's essential we use every opportunity we can to influence the shape of that system?

The BDA will submit its own response before the 31 July deadline.

Visit https://www.gov.uk/ government/consultations/ improving-dental-contracts.

\section{DENTISTS CAN NOW BLEACH DISEASED TEETH IN THE YOUNG}

General dental practitioners can now provide dental bleaching treatment for diseased teeth in patients under the age of 18, without fear of disciplinary action by the General Dental Council (GDC).

The GDC has updated its guidance after two years of campaigning by the British Society of Paediatric Dentistry (BSPD) and senior dentists. A revised position statement on the GDC's website states: 'Products containing or releasing between $0.1 \%$ and $6 \%$ hydrogen peroxide cannot be used on any person under 18 years of age except where such use is intended wholly for the purpose of treating or preventing disease.'

Dental bleaching to correct discolouration of teeth prevents the need for destructive removal of sound tooth structure. Up until now, EU legislation has effectively prohibited the use of scientifically proven, safe, low concentration bleaching products from being used in patients under age 18. Common causes of tooth discolouration include trauma, inherited dental conditions, or the swallowing of medicines while the adult teeth are developing. Opacities are among the commonest type of enamel defect.

In a recent paper Martin Kelleher, a consultant in restorative dentistry at King's College London, raised concerns regarding the psychological impacts of discoloured teeth on young patients, which can cause bullying and unhappiness. ${ }^{1}$

Dr Kelleher said of the updated guidance: 'I am glad that this unscientific position has been revised so that all patients, irrespective of age, can benefit from safe and effective treatment of their discoloured teeth in a regulated environment'.

Claire Stevens of the BSPD and consultant in paediatric dentistry said: 'We are grateful to many people for their support with our lobbying, particularly Kathy Harley, Dean of the Faculty of the Royal College of Surgeons on England, who has been instrumental in achieving the change.

'We are delighted that we can now treat [young patients] without fear of disciplinary action.'

1. Kelleher M. The law is an ass: legal and ethical issues surrounding the bleaching of young patients' discoloured teeth. Faculty Dent J 2014; 5: 56-67.

\title{
APP WILL HELP THOSE WITH DISABILITIES COMMUNICATE AT THE DENTIST
}

Scottish scientists have developed a speech app which helps children and adults with learning and/or communication disabilities prepare for a visit to the dentist.

A visit to the dentist can be an anxious time for most people. But for people with disabilities that affect communication, visits to the dentist can be particularly stressful.

The 'Stories at the dentist' communication system helps patients with a learning and/or communication disability to know what to expect when visiting the dentist and to be more involved in the decision making process concerning their care and treatment options.

Gillian Elliott, Senior Dental Officer and Specialist in Special Care Dentistry at the Community Dental Service, said: 'This app is already transforming patients' experiences in the dental setting. By improving communication, and therefore understanding, patients can become fully involved in decisions about their care. We have found that the app reduces anxiety as patients are fully prepared about what to expect at their dental visit'.

The system is the result of an 18-month long collaborative project between computing scientists, dentists and health scientists from the Universities of Dundee and St Andrews, NHS Tayside and Capability Scotland, funded by the Research Council UK Digital Economy. Patients and Community Dental staff across Tayside were involved in the design and evaluation of the app, which runs on an iPad.

The app allows a patient to interact with a customised 'story' about their next dental appointment. For some disabilities such as autism, knowing ahead of time what will happen at a dental visit is particularly important. Stories are written from the perspective of the patient and photographs of people, places and equipment help to prepare them for the visit. For some patients, explanation of different treatment options or advice on how to care for their teeth at home may be given. The app allows patients to 'tag' parts of the 'story' that make them feel anxious or they have questions about. The tagged story is used by the patient and dentist before treatment to discuss these issues.

The app serves as a tool enabling the building of rapport and understanding between a dental professional and patient making treatment less stressful for both the patients and the clinician.

The long term aim will be to develop the prototype app into a technology available as a download. 